\title{
A review of lactic acid bacteria isolated from marine animals: their species, isolation site and applications
}

\author{
${ }^{1}$ Lambuk, F., ${ }^{2 *}$ Mazlan, N., ${ }^{3}$ Thung, T.Y., ${ }^{4}$ New, C.Y., ${ }^{5}$ Rinai, K.R. and ${ }^{6}$ Son, R. \\ ${ }^{1}$ Faculty of Health and Life Sciences, Management and Science University, University Drive, Off Persiaran \\ Olahraga, Seksyen 13, 40100 Shah Alam, Selangor, Malaysia \\ ${ }^{2}$ Borneo Marine Research Institute, Universiti Malaysia Sabah, Jalan UMS, 88400 Kota Kinabalu, Sabah, \\ Malaysia. \\ ${ }^{3}$ Department of Microbiology, Biomedicine Discovery Institute, Monash University, Clayton, 3800 Australia \\ ${ }^{4}$ Go Plus Services Sdn. Bhd., 97A, Jalan BP 6/3, Bandar Bukit Puchong, 47210 Puchong, Selangor, \\ Malaysia \\ ${ }^{5}$ Institute of Bioscience, Universiti Putra Malaysia, 43400 UPM Serdang, Selangor, Malaysia \\ ${ }^{6}$ Department of Food Science, Faculty of Food Science and Technology, Universiti Putra Malaysia, 43400 \\ UPM Serdang, Selangor, Malaysia
}

\begin{abstract}
Article history:
Received: 16 February 2021

Received in revised form: 31

March 2021

Accepted: 17 June 2021

Available Online: 31 January 2022
\end{abstract}

Keywords:

Lactic acid bacteria,

Marine animal,

Probiotic,

Lactobacillus

DOI:

https://doi.org/10.26656/fr.2017.6(1).112

\begin{abstract}
Marine animals are continuously exposed to a wide range of microorganisms present in their environment. Studies indicated that the microorganisms could be found in different parts of the animal's body. There are different types of lactic acid bacteria in various isolation sites and studies have disclosed the presence of Lactobacillus, Lactococcus, Enterococcus, Streptococcus, Vagococcus, Pediococcus, Weissella, Leuconostoc, and Carnobacterium. The lactic acid bacteria (LAB) of these animals have been the subject of various studies. They were studied for their inhibitory activity against pathogens, tested for their antibiotic resistance, and searched for the production of bacteriocin which some are eventually made into commercial products. This study reviewed isolated LAB from marine animals to discover the better potential of LAB that could be implemented in different industries such as food and beverages, pharmaceutical, aquaculture, nutraceutical, and medical. This review assembled the species of LAB that were isolated from different parts of the marine animals' bodies and their application.
\end{abstract}

\section{Introduction}

Lactic acid bacteria (LAB) are microorganisms known for their benefits to mankind. They are widely distributed in carbohydrate-rich environments and typically found in decayed plants and animal matter. LAB are described as a group of Gram-positive with the shape of rods or cocci, non-spore-forming, lack of catalase activity, anaerobic or facultative aerobic, nonmotile and acid-tolerant microorganism (Florou-Paneri et al., 2013, Quinto et al., 2014, Gupta et al., 2018; Bintsis, 2018). LAB can be identified based on the morphology, their ability to ferment carbohydrates, carbon dioxide production, growth in different temperature and their ability to grow at high salt concentrations. Figure 1 shows the basic structure of a Lactic Acid Bacteria, Lactobacillus. LAB are classified into six main groups: Lactobacillus, Lactococcus, Leuconostoc, Streptococcus, Enterococcus and Pediococcus (Bennani et al., 2017).
These types of bacteria are mostly present in the human body as normal flora to the alimentary tract. Their presence ensured control over less friendly bacteria as well as giving other benefits to the host (Gupta et al., 2018). LAB have been regarded as safe food-grade microorganisms (Xu et al., 2016). They have been used as food preservatives and modifiers in flavours as well as the texture of food. LAB are also important in the fermentation of dairy products, meats, vegetables and

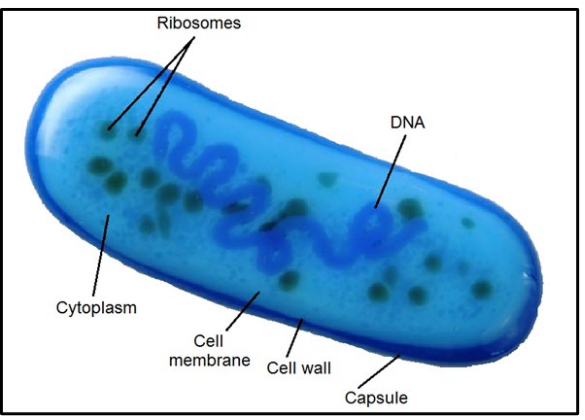

Figure 1. Structure of Lactobacillus (Anup, 2018) eISSN: 2550-2166 / C 2022 The Authors. Published by Rynnye Lyan Resources 
some traditional foods. They ferment carbohydrates and produce lactic acid as the main product of fermentation (Florou-Paneri et al., 2013).

Marine animals are adapted to live in saltwater. The animals are able to regulate salt intake, obtain oxygen and withstand the saltwater pressure (Kennedy, 2019). They live at all levels of the oceanic water column. The animals can be seen in the upper ocean, in deeper waters, and all over the ocean basins. The abundance of organisms decreases with depth (Cochran, 2014). LAB are generally considered as favourable bacteria to marine animals. They are known for their ability to act against bacterial pathogens (Merrifield et al., 2014). LAB are mostly isolated from the gastrointestinal tract (GIT) and muscle of marine animals. Variation of LAB suggests that real variation exists between marine animals' species and geographical location (Buntin et al., 2008).

\section{Lactic acid bacteria}

\subsection{Characterisation of lactic acid bacteria}

Lactate and acetate are the main products produced by LAB during carbohydrates fermentation. Previous studies proved that some LAB performed as an antagonist towards pathogenic and spoilage microorganisms (Moosavi-Nasab et al., 2014). There are two metabolic categories of LAB based on the sugar fermentation patterns: homofermentative, and heterofermentative. Figure 2 shows the metabolic pathway in LAB. Homofermentative LAB transforms almost all of the sugar into lactic acid. Meanwhile, the heterofermentative LAB does not produce lactic acid as the main product of fermentation. They may produce

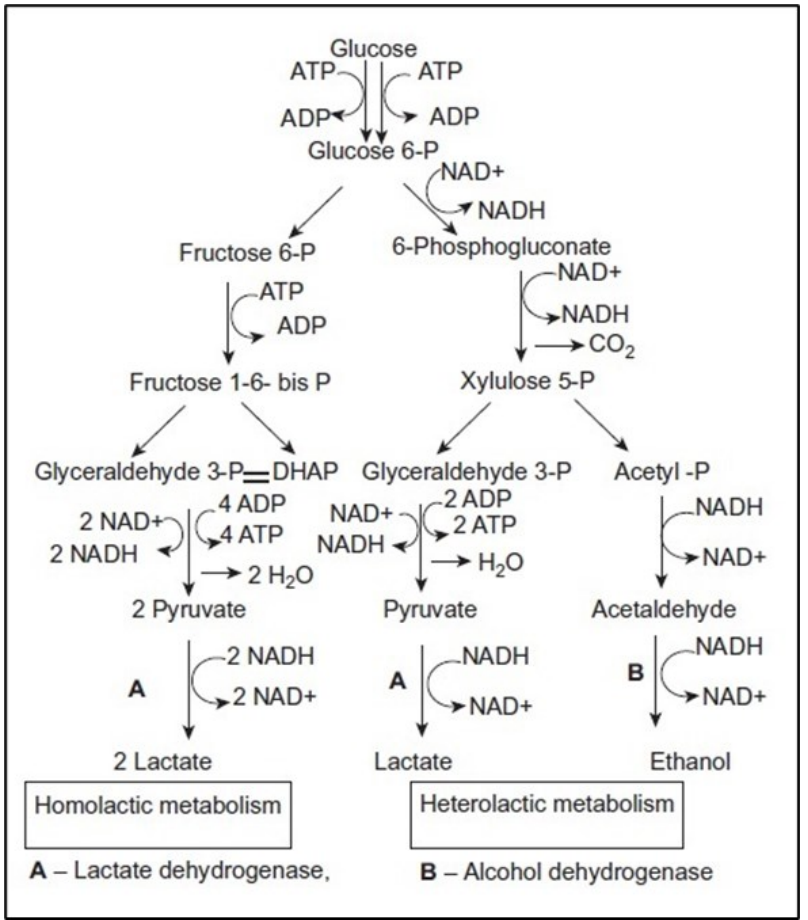

Figure 2. Metabolic pathway of Lactic Acid Bacteria (O’Bryan et al., 2015) ethanol or acetate as the by-products (Ganzle, 2015). In general, the main products of LAB fermentation include alcohol, carbon dioxide and organic acid. They also produce aromatic molecules, vitamins, or bioactive peptides (O’Bryan et al., 2015).

Some LAB possesses a limited capacity to synthesize amino acids from inorganic nitrogen sources. They depend on the presence of amino acids available in the environment or medium of growth. Proteinase and peptidase can be found as extracellular or intracellular substances of the cell. A few LAB strains are able to metabolize lipids. The strains have either intracellular or extracellular lipases. Furthermore, they perform unique fatty acid transformation reactions including isomerization, saturation, hydration and dehydration. Their metabolic activities are also able to provide health benefits to the host. Lactobacilli are able to break down cholesterol into serum lipid and this had been proved by a few studies on mice, preclinical and clinical trials (Hayek et al., 2013). Some LAB can metabolize citrate. This metabolism requires citrate transportation, citrate conversion into oxaloacetate and pyruvate. Citrate metabolism by LAB leads to the production of 4-carbon compounds, i.e diacetyl, acetoin and 2,3-butanediol. These compounds possess aromatic properties and they give a certain odour to some fermented products (Cadwallader et al., 2009).

\subsection{Application of lactic acid bacteria}

Colonization of lactic acid bacteria is important to the development and physiology of the host. In the gut, they contribute to nutrient absorption, immune response, mucosal tolerance and epithelial development. A balanced population of intestinal bacteria is crucial in maintaining the health of the host (Rungrassamee et al., 2014, Russo et al., 2015). The intestinal tract is considered to be a valuable waste and a good source for LAB isolation (Moosavi-Nasab et al., 2014).

LAB have been applied as starter cultures in foods and beverages due to their ability to improve nutritional, organoleptic, technological and shelf-life characteristics. Lactic acid is the main product of fermentation, followed by acetic acid, while LAB can also produce ethanol, bacteriocin, aroma compounds, exopolysaccharides and some enzymes (Florou-Paneri et al., 2013, Mozzi, 2016). LAB is widely used as a source of probiotics because they play important roles in the host digestive tract, improving its immune status, modulating the bacterial community, and antagonizing opportunistic pathogens. They enhance the balance of the microbial community in the intestine, confer protection against potential pathogenic bacteria, and prevent and/or cure intestinal diseases (Azat et al., 2016). Probiotics have also been 
frequently administered in aquaculture because they give out several benefits including immunological, nutritional, and environmental benefits (Sha et al., 2016). $\mathrm{LAB}$ acts as the preservation to the food by producing metabolites such as lactic acid, fatty acid and bacteriocin by inhibiting the growth of spoilage pathogenic bacteria. The compounds produced by these LAB interact with the cell membranes of the harmful pathogens and inhibit the growth (Azat et al., 2016; Gupta et al., 2018). Intestinal LAB also can produce some vitamins that are needed by the body of the host (Florou-Paneri et al., 2013). Lactobacillus spp. (L. lactis, L. plantarum, $L$. bulgaricus), Streptococcus spp. and Enterococcus spp. are able to produce folate (Masuda et al., 2014). Lactobacillus spp. has the ability to produce vitamin B12. Strains of genera Lactococcus, Lactobacillus, Enterococcus, Leuconostoc and Streptococcus are able to produce vitamin $\mathrm{K}$. LAB release a variety of enzymes into the gastrointestinal tract. Enzymes produced by the LAB during food fermentation are amylases and peptidases. Amylases are being used in sourdough technology and peptidases are used for cheese making. $\mathrm{LAB}$ are also important in winemaking. The bacteria grow in wine during malolactic fermentation, following alcoholic fermentation. These secondary modifications improve the taste and flavour of wine (Florou-Paneri et al., 2013).

\section{Lactic acid bacteria isolated from marine animals}

\subsection{Shellfish}

LAB isolated from the gastrointestinal tract of wild shrimp was revealed to have high prevalence and diversity. The LAB isolated from the wild shrimp also possesses inhibitory properties towards Vibrio harveyi. Coccoid LAB is the most prevalent $\mathrm{LAB}$ in the gastrointestinal tract of aquatic animals (Kongnum et al., 2012). Table 1 shows the LAB isolated from various species of shellfish. Streptococcus phocae isolated from Indian white shrimp by Kanmani and colleagues (2010) has the ability to restrain mortality and improve the survival rate of the shrimp. Lactococcus lactis isolated from kuruma shrimp (Marsupenaeus japonicus) was claimed to have the potential in controlling and preventing Vibrio penaeicida infection (Maeda et al., 2014). Samples taken from muscle and intestine of shrimp collected from Tunisia showed the presence of Lb. paracasei (Boulares et al., 2011). Microbiota that inhabits the intestine of wild-caught and domesticated giant tiger prawn (Penaeus monodon) were compared by Rungrassamee and team (2014). The study showed the presence of Lactobacillus sp., Lactococcus sp. and Pediococcus sp. in wild-caught giant tiger prawns. Out of the 24 isolates from the GIT of black tiger shrimp
(Penaeus monodon) and ornate spiny lobster (Panulirus ornatus), 8.3\% was detected as Enterococcus faecalis. This strain showed strong antimicrobial activity against Proteus sp., Proteus mirabilis and E. coli. However, the antimicrobial activity of its bacteriocins lost completely after incubation at $60^{\circ} \mathrm{C}$ for 30 mins (Nguyen et al., 2014). Azahar et al (2018) had isolated 14 probiotic strains from GIT of prawn (Macrobrachium rosenbergii). Molecular identification using 16s rRNA genes sequences identified them as E. faecalis, Lc. lactis and Lc. garvieae. Three of the 14 strains showed antimicrobial activities towards $V$. parahaemolyticus, $V$. alginolyticus and Aeromonas hydrophila. However, the authors did not mention the specific strains which possess growth inhibition towards the pathogens.

Lactobacillus spp. were isolated from the oyster (Crassostrea gigas) by Lee and colleagues (2010) and was expected to be more adaptable to marine aquaculture conditions compared to freshwater animals. Kang et al. (2016) claimed that Lb. rhamnosus MH22 exhibited relatively high antagonism to the two strains of Vibrio spp. Lb. plantarum isolated from the muscle of shellfish; oyster (Crassostrea gigas) and shortnek clam (Tapes philippinarum) showed inhibitory activity against pathogens including E. coli, Edwardsiella tarda, Staphylococcus aureus, Salmonella enterica serovar Enteriditis, Streptococcus inae, S. enterica serovar Typhimurium, $V$. ichthyoenteri and $V$. parahaemolyticus (Kang et al., 2016). Meanwhile, Lactobacillus paracasei ssp. paracasei was successfully isolated from the gastrointestinal tract of abalone (Holiotis asinina). This species was discovered to be able to inhibit the growth of enteropathogenic bacteria (E. coli, Bacillus cereus and $S$. aureus) and able to grow in acidic conditions and tolerant of bile during $24 \mathrm{hrs}$ incubation (Sarkono et al., 2010).

Furthermore, the intestinal content of clam (Meretrix lamarckii) may harbour different Lc. lactis subsp. lactis and Lc. lactis subsp. cremoris strains as reported by Itoi et al. (2013). The clam inhabits the intertidal zone with the changes of osmotic pressure, dissolved oxygen and temperature. The changes would also force the LAB to adapt to the condition. Leuconostoc pentosus and E. faecium was isolated from the whole body without a shell of oyster (Crassostrea gigas). Enterococcus faecium was found to be resistant to 10 types of antibiotics. Probiotic strains with antibiotic resistance would be captivating if the probiotic is administered during antibiotic treatment (Kang et al., 2017).

Kim and colleagues (2017) studied the influence of season and changes in water temperature on the microbiota of swimming crab (Portunus trituberculatus). 
Table 1. Lactic acid bacteria isolated from shellfish

\begin{tabular}{|c|c|c|c|c|}
\hline Marine organism & Location & Lactic Acid Bacteria & $\begin{array}{c}\text { Part of } \\
\text { isolation }\end{array}$ & References \\
\hline $\begin{array}{l}\text { Indian white shrimp } \\
\text { (Penaeus indicus) }\end{array}$ & ni* & Streptococcus phocae & GIT & $\begin{array}{l}\text { Kanmani et al. } \\
(2010)\end{array}$ \\
\hline $\begin{array}{l}\text { Kuruma shrimp } \\
\text { (Marsupenaeus } \\
\text { japonicus) }\end{array}$ & $\begin{array}{l}\text { Kanmon Strait, } \\
\text { Fukuoka and } \\
\text { Tachibana Bay, } \\
\text { Nagasai, Japan }\end{array}$ & $\begin{array}{l}\text { Enterococcus faecalis, E. faecium, E. } \\
\text { pseudovium, E. raffinosus, Lactobacillus sp., } \\
\text { Lb. plantarum, Lb. nagelii, Lactococcus } \\
\text { garvieae, L. lactis, Pediococcus pentosaceus, } \\
\text { Vagococcus campiphilus, Vc. Fluvialis, E. } \\
\text { pallens, Lb. amylophilus }\end{array}$ & GIT & $\begin{array}{l}\text { Maeda et al. } \\
(2014)\end{array}$ \\
\hline
\end{tabular}

\begin{tabular}{|c|c|c|c|c|}
\hline $\begin{array}{l}\text { Giant tiger prawn } \\
\text { (Penaeus monodon) }\end{array}$ & $\begin{array}{l}\text { Andaman Sea, } \\
\text { Thailand }\end{array}$ & $\begin{array}{l}\text { Lactobacillus sp., Lactococcus sp., } \\
\text { Pediococcus } \mathrm{sp} .\end{array}$ & GIT & $\begin{array}{l}\text { Rungrassamee } \text { et } \\
\text { al. (2014) }\end{array}$ \\
\hline $\begin{array}{l}\text { Banana shrimp } \\
\text { (Fenneropenaeus } \\
\text { merguiensis) }\end{array}$ & $\begin{array}{l}\text { Songkhla province, } \\
\text { Thailand }\end{array}$ & LAB & GIT & $\begin{array}{l}\text { Kongnum et al. } \\
(2012)\end{array}$ \\
\hline $\begin{array}{l}\text { Swimming crab } \\
\text { (Callinectes sp.) }\end{array}$ & Lagos, Nigeria & Streptococcus agalactie & GIT & $\begin{array}{l}\text { Uaboi-Egbenni et } \\
\text { al. (2010) }\end{array}$ \\
\hline $\begin{array}{l}\text { Blue swimming crab } \\
\text { (Portunus pelagicus) }\end{array}$ & $\begin{array}{l}\text { Strait of Tebrau, } \\
\text { Johor, Malaysia }\end{array}$ & $\begin{array}{l}\text { Lactobacillus plantarum, Lb. salivarius, Lb. } \\
\text { rhamnosus, Weissella confusa and } W . \text { cibaria }\end{array}$ & GIT & $\begin{array}{l}\text { Talpur et al. } \\
\text { (2012) }\end{array}$ \\
\hline $\begin{array}{l}\text { Swimming crab } \\
\text { (Portunus } \\
\text { trituberculatus) }\end{array}$ & Yellow Sea, Korea & $\begin{array}{l}\text { Carnobacterium, Lactococcus, Streptococcus } \\
\text { and Vagococcus }\end{array}$ & Whole body & Kim et al. (2017) \\
\hline $\begin{array}{l}\text { Oyster (Crassostrea } \\
\text { gigas) }\end{array}$ & $\begin{array}{l}\text { Taean coast, South } \\
\text { Korea }\end{array}$ & $\begin{array}{l}\text { Lactobacillus pentosus, Enterococcus } \\
\text { faecium }\end{array}$ & $\begin{array}{l}\text { Whole body } \\
\text { without shell }\end{array}$ & $\begin{array}{l}\text { Kang et al. } \\
\text { (2017) }\end{array}$ \\
\hline $\begin{array}{l}\text { Corb shell (Cyclina } \\
\text { sinensis) }\end{array}$ & West Sea, Korea & Lactobacillus sp. & Muscle & $\begin{array}{l}\text { Kang et al. } \\
\text { (2016) }\end{array}$ \\
\hline $\begin{array}{l}\text { Mussel (Mytilus } \\
\text { edulis) }\end{array}$ & West Sea, Korea & Lactobacillus sp. & Muscle & $\begin{array}{l}\text { Kang et al. } \\
\text { (2016) }\end{array}$ \\
\hline $\begin{array}{l}\text { Surf clam (Mactra } \\
\text { veneriformis } \text { Reeve) }\end{array}$ & West Sea, Korea & Lactobacillus sp. & Muscle & $\begin{array}{l}\text { Kang et al. } \\
\text { (2016) }\end{array}$ \\
\hline $\begin{array}{l}\text { Oyster (Crassostrea } \\
\text { gigas) }\end{array}$ & West Sea, Korea & Lactobacillus sp. and Lb. plantarum & Muscle & $\begin{array}{l}\text { Kang et al. } \\
\text { (2016) }\end{array}$ \\
\hline $\begin{array}{l}\text { Shortnek clam (Tapes } \\
\text { philippinarum) }\end{array}$ & West Sea, Korea & Lactobacillus plantarum & Muscle & $\begin{array}{l}\text { Kang et al. } \\
\text { (2016) }\end{array}$ \\
\hline $\begin{array}{l}\text { Spiny top shell } \\
\text { (Batillus cornutus) }\end{array}$ & West Sea, Korea & Lactobacillus sp. & Muscle & $\begin{array}{l}\text { Kang et al. } \\
(2016)\end{array}$ \\
\hline $\begin{array}{l}\text { Mud crab (Scylla } \\
\text { paramamosain) }\end{array}$ & $\begin{array}{l}\text { Southern Chinese } \\
\text { coasts, China }\end{array}$ & Lactobacillus, Lactococcus & GIT & Wei et al. (2019) \\
\hline $\begin{array}{l}\text { Mud crab (Scylla } \\
\text { paramamosain) }\end{array}$ & Shantou, China & $\begin{array}{l}\text { Enterococcus faecalis, Pediococcus } \\
\text { pentosaceus }\end{array}$ & GIT & $\begin{array}{l}\text { Yang et al. } \\
(2019)\end{array}$ \\
\hline $\begin{array}{l}\text { Clam (Meretrix } \\
\text { lamarckii) }\end{array}$ & Ibaraki, Japan & $\begin{array}{l}\text { Lb. curvatus, Lb. plantarum, Lc. lactis ssp } \\
\text { cremoris, Pediococcus pentosaceus, Lc. lactis } \\
\text { ssp. lactis }\end{array}$ & GIT & Itoi et al. (2013) \\
\hline $\begin{array}{l}\text { Prawn } \\
\text { (Macrobrachium } \\
\text { rosenbergii) }\end{array}$ & $\begin{array}{l}\text { Kuala Terengganu, } \\
\text { Terengganu, Malaysia }\end{array}$ & $\begin{array}{l}\text { Enterococcus faecalis, Lc. lactis and Lc. } \\
\text { garvieae }\end{array}$ & GIT & $\begin{array}{l}\text { Azahar et al. } \\
(2018)\end{array}$ \\
\hline Shrimp & Tunisia & Lb. paracasei & $\begin{array}{l}\text { Muscle and } \\
\text { intestine }\end{array}$ & $\begin{array}{l}\text { Boulares et al. } \\
\text { (2011) }\end{array}$ \\
\hline $\begin{array}{l}\text { Abalone (Holiotis } \\
\text { asinina) }\end{array}$ & Lombok, Indonesia & Lb. paracasei ssp. paracasei & GIT & $\begin{array}{l}\text { Sarkono et al. } \\
\text { (2010) }\end{array}$ \\
\hline $\begin{array}{l}\text { Oyster (Crassostrea } \\
\text { gigas) }\end{array}$ & $\begin{array}{l}\text { Geunso bay of Taean, } \\
\text { South Korea }\end{array}$ & $\begin{array}{l}\text { Lb. paracasei, Lb. plantarum, Lb. johnsonii, } \\
\text { Lb. rhamnosus, Lb. parabuchneri, Lb. } \\
\text { pentosus, Lb. paraplantarum }\end{array}$ & $\begin{array}{l}\text { Whole body } \\
\text { without shell }\end{array}$ & Lee et al. (2010) \\
\hline $\begin{array}{l}\text { Black tiger shrimp } \\
\text { (Penaeus monodon) }\end{array}$ & $\begin{array}{l}\text { Khanh Hoa province, } \\
\text { Vietnam }\end{array}$ & Enterococcus faecalis & GIT & $\begin{array}{l}\text { Nguyen et al. } \\
(2014)\end{array}$ \\
\hline $\begin{array}{l}\text { Ornate spiny lobster } \\
\text { (Panulirus ornatus) }\end{array}$ & $\begin{array}{l}\text { Khanh Hoa province, } \\
\text { Vietnam }\end{array}$ & Enterococcus faecalis & GIT & $\begin{array}{l}\text { Nguyen et al. } \\
(2014)\end{array}$ \\
\hline
\end{tabular}
(Panulirus ornatus)

* ni - no further information, GIT - gastrointestinal tract, E. - Enterococcus, Lb. - Lactobacillus, Lc. - Lactococcus, Vc. Vagococcus, W. - Weissella 
They isolated Streptococcus, Lactococcus, Carnobacterium and Vagococcus from the whole body of the swimming crab and the isolated LAB were the dominant genera in spring crab. Yang et al. (2019) isolated E. faecalis and P. pentosaceus from GIT of mud crab (Scylla paramamosain). They found E. faecalis and $P$. pentosaceus exhibited a strong growth inhibition to $A$. hydrophila, Beta Streptococcus, $S$. aureus and $V$. alginolyticus. A study conducted by Talpur and colleagues (2012) indicated that $L b$. plantarum, $L b$. salivarius and $L b$. rhamnosus isolated from the gastrointestinal tract of blue swimming crab (Portunus pelagicus)improved the survival of $P$. pelagicus larvae. GIT of female mud crab (Scylla paramamosain) assembled $0.37 \%$ abundance Lactobacillus genus. Genus of Lactococcus and Lactobacillus were found in GIT of male samples with a relative abundance of $0.7 \%$ and $1.10 \%$ respectively (Wei et al., 2019). S. agalactiae was isolated from GIT of swimming crab (Callinectes sp.). The swimming crab was collected from the open lagoon at $28^{\circ} \mathrm{C}$ and from the market samples at $0^{\circ} \mathrm{C}$. The study showed the least hemolytic values compared to other isolated bacteria. This strain was previously belonging to pyrogenic mastitis in cows (Uaboi-Egbenni et al., 2010).

\subsection{Finfish}

LAB has gained attention with respect to its beneficial effects on fish health. LAB play important role in the fish GIT. The LAB is able to stimulate the fish GI development, digestive function, mucosal tolerance, stimulate immune response and improve disease resistance (Ringo et al., 2018). Wild fish could be a substantial source of LAB that could be suitable for use as probiotics in feed for fish (Kim et al., 2013). Table 2 shows the LAB isolated from finfish. A study conducted by Salas-Leiva and colleagues (2017) reported that they had isolated Carnobacterium, $C$. divergen, Lactobacillus, Streptococcus, Vagococcus, and Weissella from the intestine of fine flounder (Paralichthys adspersus). Boulares and team (2011) had isolated LAB from muscle and intestine of eight types of marine fish. Lactobacillus was mostly found genus in fish followed by Lactococcus. The team also isolated $L b$. paracasei from red mullet, Lb. brevaris from whiting and Leu. citream from pageot. The most dominant phylum isolated from Atlantic salmon (Salmo salar) was Firmicutes. Carnobacterium, C. divergen, Lactobacillus, Lactococcus, Streptococcus were found in the gastrointestinal tract of the fish (Dehler et al., 2017). A study conducted by Svanevik and Lunestad (2011) was to examine the microorganisms obtained from the gill, skin and GIT of Atlantic mackerel (Scomber scombrus). As the result, they found two species of LAB which were Vagococcus sp. (isolated from GIT) and V. carniphilus (isolated from gill, skin and GIT).

Lactobacillus. buchneri, Lc. lactis, Lb. acidophilus, $L b$. fermentum, and $S$. salivarius isolated from the intestine of Narrow-barred Spanish mackerel (Scomberomorus commerson) showed different degrees of inhibitory activity. It was probably due to the synergistic effect of both bacteria and antimicrobial substances, hence enhancing their antimicrobial effect against Listeria innocua (Moosavi-Nasab et al., 2014). S. parauberis strain maris rubric was identified from broomtail wrasse (Cheilinus lunulatus). It is known for causing bovine mastitis and this type of LAB was tested to be resistant to oxytetracycline, baytril (enofloxacin) and doxycycline. Streptococcus iniae was isolated from the goatfish (Parupeneus sp.), grouper (Epinephelus fasciatus), and rabbitfish (Siganus rivulatus). This strain was tested to be sensitive to oxytetracycline, chloramphenicol, florfenicol, baytril (enrofloxacin), doxycycline, sulphamethoxazole/trimethoprim and clindamycin (Ucko et al., 2013). The LAB isolated from the gut of Indian mackerel (Rastrelliger kanagurta) were identified as $L b$. plantarum, $L b$. viridiscens, $L b$. bulgaricus, and $L b$. brevis. From the isolated LAB, $L b$. plantarum was found to possess good antibacterial activity (Ghosh et al., 2014). Enterococcus durans was isolated from the viscera of five species of fish from the Mediterranean coast, Tunisia. E. faecium was the only LAB found from red mullet (Mullus surmutelus) and $L c$. lactis from picarel (Spicara smaris). Lc. lactis from picarels showed a broader inhibitory spectrum and displayed important inhibitory activity against Saccharomyces cerevisiae and Candida pseudotropicalis. A bacteriocin was purified from $E$. durans (isolated from Pandora). The molecular mass of the purified peptide was $6316.89 \pm 0.64 \mathrm{Da}$ (Migaw et al., 2013).

Belfiore and colleagues (2010) also isolated four species of Leuconostoc from anchovy (Engraulis anchoita). They suggested that the generated amino acid from fish would be transformed into aroma compounds by $L c$. mesenteroides subsp. dextranicum, Lc. carnosum and $L c$. mesenteroides subsp. mesenteroides. An antiListeria compound was also detected for Leuc. mesenteroides. Two species of LAB; $L b$. casei and $L b$. plantarum were selected from 84 isolated lactobacilli from the intestine of Persian sturgeon (Acipenser persicus) and Beluga (Huso huso). From the study, both species were able to produce antibacterial substances. They have a high inhibitory potential against Listeria monocytogenes (Ghanbari et al., 2013). A total of 13 marine fishes obtained from Cantabrian Sea Coast, Gijou, Spain indicated that the main LAB was Lc. lactis subsp. lactis. The entire five isolated LAB displayed a 
Table 2. Lactic acid bacteria isolated from finfish

\begin{tabular}{|c|c|c|c|c|}
\hline Marine Organism & Location & Lactic Acid Bacteria & $\begin{array}{l}\text { Part of } \\
\text { Isolation }\end{array}$ & References \\
\hline $\begin{array}{l}\text { Persian sturgeon (Acipenser } \\
\text { persicus), } \\
\text { Beluga (Huso huso) }\end{array}$ & $\begin{array}{l}\text { Caspian Sea, } \\
\text { Iran }\end{array}$ & Lactobacillus casei, Lb. plantarum & Intestine & $\begin{array}{l}\text { Ghanbari et al. } \\
\text { (2013) }\end{array}$ \\
\hline Sardine (Sardina pilchardus) & ni* & $\begin{array}{l}\text { Lactobacillus homohiochii, } L b \text {. } \\
\text { farciminis }\end{array}$ & Viscera & $\begin{array}{l}\text { Poffo et al. } \\
(2011)\end{array}$ \\
\hline Leatherjacket (Oligoplites saliens) & $\mathrm{ni}^{*}$ & Lb. intestinalis & Viscera & Poffo et al. \\
\hline Sardine & Tunisia & $\begin{array}{l}\text { Lc. lactis, Carnobacterium } \\
\text { divergens, Carnobacterium }\end{array}$ & $\begin{array}{l}\text { Muscle and } \\
\text { intestine }\end{array}$ & $\begin{array}{l}\text { Boulares et al. } \\
\text { (2011) }\end{array}$ \\
\hline Whiting & Tunisia & Lb. brevis & $\begin{array}{l}\text { Muscle and } \\
\text { intestine }\end{array}$ & Boulares et al. \\
\hline Sole & Tunisia & $\begin{array}{l}\text { Leuconostoc mesenteroides, } L b \text {. } \\
\text { brevis }\end{array}$ & $\begin{array}{l}\text { Muscle and } \\
\text { intestine }\end{array}$ & $\begin{array}{l}\text { Boulares et al. } \\
(2011)\end{array}$ \\
\hline Red mullet & Tunisia & Lb. paracasei & $\begin{array}{l}\text { Muscle and } \\
\text { intestine }\end{array}$ & Boulares et al. \\
\hline Mullet & Tunisia & $\begin{array}{l}\text { Lc. lactis, Leuconostoc } \\
\text { mesenteroides, Lb. plantarum, Lb. }\end{array}$ & $\begin{array}{l}\text { Muscle and } \\
\text { intestine }\end{array}$ & $\begin{array}{l}\text { Boulares et al. } \\
\text { (2011) }\end{array}$ \\
\hline Sea bream & Tunisia & $\begin{array}{l}\text { Lc. lactis, Leu. Mesenteroides, Lb. } \\
\text { plantarum, Lb. paracasei, Lb. } \\
\text { brevis, Carnobacterium piscicola, } \\
\text { Lb. delbruekeii }\end{array}$ & $\begin{array}{l}\text { Muscle and } \\
\text { intestine }\end{array}$ & $\begin{array}{l}\text { Boulares et al. } \\
\text { (2011) }\end{array}$ \\
\hline Atlantic salmon (Salmo salar) & $\begin{array}{l}\text { West coast of } \\
\text { Scotland }\end{array}$ & $\begin{array}{l}\text { Carnobacterium, C. divergen, } \\
\text { Lactobacillus, Lactococcus, } \\
\text { Streptococcus }\end{array}$ & GIT & $\begin{array}{l}\text { Dehler et al. } \\
(2017)\end{array}$ \\
\hline $\begin{array}{l}\text { Fine flounder (Paralichthys } \\
\text { adspersus) }\end{array}$ & $\begin{array}{l}\text { Region of } \\
\text { Coquimbo, } \\
\text { Chile }\end{array}$ & $\begin{array}{l}\text { Carnobacterium, C. divergen, } \\
\text { Lactobacillus, Streptococcus, } \\
\text { Vagococcus, Weissella }\end{array}$ & Intestine & $\begin{array}{l}\text { Salas-Leiva et } \\
\text { al. (2017) }\end{array}$ \\
\hline $\begin{array}{l}\text { Broomtail wrasse (Cheilinus } \\
\text { lunulatus) }\end{array}$ & Eilat, Israel & $\begin{array}{l}\text { Streptococcus parauberis strain } \\
\text { marin rubri }\end{array}$ & $\begin{array}{l}\text { Spleen, } \\
\text { kidney, } \\
\text { blood }\end{array}$ & $\begin{array}{l}\text { Ucko et al. } \\
\text { (2014) }\end{array}$ \\
\hline Goatfish (Parupeneus sp.) & Eilat, Israel & Streptococcus iniae & $\begin{array}{l}\text { Spleen, } \\
\text { kidney, } \\
\text { blood }\end{array}$ & $\begin{array}{l}\text { Ucko et al. } \\
\text { (2014) }\end{array}$ \\
\hline Grouper (Epinephelus fasciatus) & Eilat, Israel & Streptococcus iniae & $\begin{array}{l}\text { Spleen, } \\
\text { kidney, } \\
\text { blood }\end{array}$ & $\begin{array}{l}\text { Ucko et al. } \\
\text { (2014) }\end{array}$ \\
\hline Rabbitfish (Siganus rivulatus) & Eilat, Israel & Streptococcus iniae & $\begin{array}{l}\text { Spleen, } \\
\text { kidney, } \\
\text { blood }\end{array}$ & $\begin{array}{l}\text { Ucko et al. } \\
\text { (2014) }\end{array}$ \\
\hline $\begin{array}{l}\text { Indian mackerel (Rastrelliger } \\
\text { kanagurta) }\end{array}$ & $\begin{array}{l}\text { Ernakulan, } \\
\text { Kerala, India }\end{array}$ & $\begin{array}{l}\text { Lb. plantarum, Lb. viridiscens, } L b . \\
\text { bulgaricus, Lb. brevis }\end{array}$ & GIT & $\begin{array}{l}\text { Ghosh et al. } \\
(2014)\end{array}$ \\
\hline $\begin{array}{l}\text { Olive flounder (Paralichthyls } \\
\text { olivaceus) }\end{array}$ & $\begin{array}{l}\text { Wando, South } \\
\text { Korea }\end{array}$ & $\begin{array}{l}\text { Streptococcus salivarius, } \\
\text { Lactococcus lactis, Lc. } \\
\text { chungangensis, Enterococcus } \\
\text { durans, Leuconostoc lactis, } \\
\text { Weissella cibaria, } W . \\
\text { thailandensis, Lb. brevis, Lb. } \\
\text { crustorum, Lb. sakei, Pediococcus } \\
\text { pentosaceus, Lb. oligofermentans }\end{array}$ & $\begin{array}{l}\text { Mucus of } \\
\text { hindgut }\end{array}$ & $\begin{array}{l}\text { Kim et al. } \\
(2013)\end{array}$ \\
\hline Anchovy (Engraulis anchoita) & $\begin{array}{l}\text { Mar del Plata, } \\
\text { Argentina }\end{array}$ & $\begin{array}{l}\text { Leuconostoc mesenteroides ssp. } \\
\text { mesenteroide, Leu. mesenteroides } \\
\text { ssp. dextranicum, Leu. } \\
\text { mesenteroides ssp. cremoris, Leu. } \\
\text { mesenteroides ssp. carnosum }\end{array}$ & GIT & $\begin{array}{l}\text { Belfiore et al. } \\
(2010)\end{array}$ \\
\hline
\end{tabular}

* ni - no further information, Lb. - Lactobacillus, Lc. - Lactococcus, Leu. - Leuconostoc, C. - Carnobacterium, W. - Weissella, S. - Streptococcus, E. - Enterococcus, GIT - gastrointestinal tract 
Table 2 (Cont.). Lactic acid bacteria isolated from finfish

\begin{tabular}{|c|c|c|c|c|}
\hline Marine Organism & Location & Lactic Acid Bacteria & $\begin{array}{c}\text { Part of } \\
\text { Isolation }\end{array}$ & References \\
\hline $\begin{array}{l}\text { White trevally (Pseducaranx } \\
\text { dentex) }\end{array}$ & $\begin{array}{l}\text { Moreton Bay, } \\
\text { Sunshine Coast } \\
\text { and Gold Coast, } \\
\text { Queensland, } \\
\text { Australia }\end{array}$ & $\begin{array}{l}\text { Streptococcus uberis, S. equinus, } \\
\text { S. constellatus, Enterococcus } \\
\text { faecium, E. avium, Vagococcus } \\
\text { fluvialis }\end{array}$ & $\begin{array}{l}\text { Muscle, gill, } \\
\text { gut }\end{array}$ & $\begin{array}{l}\text { Al Bulushi et } \\
\text { al. }(2010)\end{array}$ \\
\hline Silver seabream (Pagrus auratus) & $\begin{array}{l}\text { Moreton Bay, } \\
\text { Sunshine Coast } \\
\text { and Gold Coast, } \\
\text { Queensland, } \\
\text { Australia }\end{array}$ & $\begin{array}{l}\text { S. uberis, } S . \text { equinus, } \text { S. salivarius, } \\
\text { S. constellatus, E. faecium, } \\
\text { Aerococcus spp. }\end{array}$ & $\begin{array}{l}\text { Muscle, gill, } \\
\text { gut }\end{array}$ & $\begin{array}{l}\text { Al Bulushi et } \\
\text { al. (2010) }\end{array}$ \\
\hline $\begin{array}{l}\text { Flathead grey mullet (Mugil } \\
\text { cephalus) }\end{array}$ & $\begin{array}{l}\text { Moreton Bay, } \\
\text { Sunshine Coast } \\
\text { and Gold Coast, } \\
\text { Queensland, } \\
\text { Australia }\end{array}$ & $\begin{array}{l}\text { S. uberis, S. equinus, s. salivarius, } \\
\text { S. constellatus, E. faecium, E. } \\
\text { avium, Aerococcus spp., } \\
\text { Carnobacterium spp. }\end{array}$ & $\begin{array}{l}\text { Muscle, gill, } \\
\text { gut }\end{array}$ & $\begin{array}{l}\text { Al Bulushi et } \\
\text { al. (2010) }\end{array}$ \\
\hline $\begin{array}{l}\text { Narrow-barred Spanish mackerel } \\
\text { (Scomberomorus commerson) }\end{array}$ & Persian Gulf & $\begin{array}{l}\text { Lb. buchneri, Lc. lactis, Lb. } \\
\text { acidophilus, Lb. fermentum, } \\
\text { Streptococcus salivarius }\end{array}$ & Intestine & $\begin{array}{l}\text { Moosavi-Nasab } \\
\text { et al. (2014) }\end{array}$ \\
\hline $\begin{array}{l}\text { Caspian sea golden grey mullet } \\
\text { (Liza aurata) }\end{array}$ & $\begin{array}{l}\text { Caspian Sea, } \\
\text { Northern Iran } \\
\end{array}$ & LAB & Muscle & $\begin{array}{l}\text { Bahmani et al. } \\
(2013)\end{array}$ \\
\hline $\begin{array}{l}\text { Atlantic mackerel (Scomber } \\
\text { scombrus) }\end{array}$ & Norwegian Sea & $\begin{array}{l}\text { Vagococcus sp. and Vagococcus } \\
\text { carniphilus }\end{array}$ & $\begin{array}{l}\text { Gill, skin and } \\
\text { gut }\end{array}$ & $\begin{array}{l}\text { Svanevik and } \\
\text { Lunestad } \\
(2011)\end{array}$ \\
\hline Sea bass & Tunisia & $\begin{array}{l}\text { Lc. lactis, Leu. mesenteroides, Lb. } \\
\text { plantarum, Lb. paracasei, Lb. } \\
\text { brevis, Lb. acidophilus }\end{array}$ & $\begin{array}{l}\text { Muscle and } \\
\text { intestine }\end{array}$ & $\begin{array}{l}\text { Boulares et al. } \\
\text { (2011) }\end{array}$ \\
\hline Pageot & Tunisia & Lb. acidophilus & $\begin{array}{l}\text { Muscle and } \\
\text { intestine }\end{array}$ & $\begin{array}{l}\text { Boulares et al. } \\
(2011)\end{array}$ \\
\hline Picarel (Spicara smaris) & $\begin{array}{l}\text { Mediterranean } \\
\text { coast, Tunisia }\end{array}$ & Lc. lactis & Viscera & Migaw et al. \\
\hline Red mullet (Mullus surmutelus) & $\begin{array}{l}\text { Mediterranean } \\
\text { coast, Tunisia }\end{array}$ & Enterococcus faecium & Viscera & Migaw et al. \\
\hline $\begin{array}{l}\text { Horse mackerel (Trachurus } \\
\text { trachurus) }\end{array}$ & $\begin{array}{l}\text { Mediterranean } \\
\text { coast, Tunisia }\end{array}$ & Enterococcus durans & Viscera & $\begin{array}{l}\text { Migaw et al. } \\
(2013)\end{array}$ \\
\hline Bogue (Boops boops) & $\begin{array}{l}\text { Mediterranean } \\
\text { coast, Tunisia }\end{array}$ & Enterococcus durans & Viscera & $\begin{array}{l}\text { Migaw et al. } \\
(2013)\end{array}$ \\
\hline Stripped mullet (Mugil cephalus) & $\begin{array}{l}\text { Mediterranean } \\
\text { coast, Tunisia }\end{array}$ & Enterococcus durans & Viscera & $\begin{array}{l}\text { Migaw et al. } \\
(2013)\end{array}$ \\
\hline Pandora (Pagellus erythrinus) & $\begin{array}{l}\text { Mediterranean } \\
\text { coast, Tunisia }\end{array}$ & Enterococcus durans & Viscera & $\begin{array}{l}\text { Migaw et al. } \\
(2013)\end{array}$ \\
\hline $\begin{array}{l}\text { Atlantic mackerel (Scomber } \\
\text { scombrus) }\end{array}$ & $\begin{array}{l}\text { Mediterranean } \\
\text { coast, Tunisia }\end{array}$ & Enterococcus durans & Viscera & $\begin{array}{l}\text { Migaw et al. } \\
(2013)\end{array}$ \\
\hline $\begin{array}{l}\text { Ballan wrasse (Labrus bergyta), } \\
\text { White seabream (Diplodus sargus } \\
\text { sargus), Peter's fish (Zeus faber), } \\
\text { European conger (Conger } \\
\text { conger), Red scorpionfish } \\
\text { (Scorpeana scrofa), Gray mullet } \\
\text { (Mugil cephalus), Zebra sea } \\
\text { bream (Diplodus cervinus } \\
\text { cervinus), Dreamfish (Sarpa } \\
\text { salpa), Small-spotted catshark } \\
\text { (Scyliorhinus canicula), } \\
\text { Triggerfish (Balistes capriscus) } \\
\text { Cuckoo wrasse (Labrus mixtus) }\end{array}$ & $\begin{array}{l}\text { Cantabrian Sea } \\
\text { Coast, Gijon, } \\
\text { Spain }\end{array}$ & $\begin{array}{l}\text { Lactobacillus plantarum, } \\
\text { Leuconostoc mesenteroides, } \\
\text { Enterococcus faecalis, E. } \\
\text { gallinarum, Lactococcus lactis ssp. } \\
\text { lactis }\end{array}$ & GIT & $\begin{array}{l}\text { Alonso et al. } \\
(2018)\end{array}$ \\
\hline
\end{tabular}

* ni - no further information, Lb. - Lactobacillus, Lc. - Lactococcus, Leu. - Leuconostoc, C. - Carnobacterium, W. - Weissella, S. - Streptococcus, E. - Enterococcus, GIT - gastrointestinal tract 
broad-spectrum antibacterial activity against $V$. harveyi, $V$. splendidus, and Photobacterium damselae (Alonso et al., 2018).

Al Bulushi et al. (2010) conducted a study by storing the white trevally (Pseducaranx dentex), silver seabream (Pagrus auratus) and flathead grey mullet (Mugil cephalus) at $25^{\circ} \mathrm{C}$ for 15 hours. They mentioned that the highest frequency of LAB was found in the gills of the fish and the lowest in the gut. From that, the frequencies of $S$. uberis and E. faecium were found the most at 15 hours of storage. Bahmani et al. (2014) studied the effect of delayed icing on the quality of mullet. They investigated the total viable count of $\mathrm{LAB}$ and Enterobacteriaceae. From their study, the growth rate of the total viable count population of bacteria decreased at the end of storage. They suggested that the bacteria might be inhibited or killed with the more production of natural preservatives by LAB such as short-chain fatty acid and bacteriocins. This condition may help in maintaining an appropriate $\mathrm{pH}$ and protect against pathological changes in fish during storage. Nine LAB were isolated from sardine (Sardina pilchardus) and leatherjacket (Oligoplites saliens). Lb. homohiochii and $L b$. farciminis were detected from viscera of sardine and Lb. intestinalis was obtained from viscera of leatherjacket. Further study showed that Lb. homohiochii grew optimally at $40^{\circ} \mathrm{C}$, meanwhile $L b$. intestinalis grew optimally at $30^{\circ} \mathrm{C}$ (Poffo et al., 2011).

\subsection{Invertebrate marine organism}

Table 3 shows LAB isolated from invertebrate marine organisms. A study conducted by Iehata et al. (2013) discovered that there were different bacterial communities and bacterial nutritional enzyme activity between both female and male Chilean octopus (Octopus mimus Gould). They suggested the possibility of different feeding habits and the formation of the bacterial community could reflect the environment of the host digestive tract. From this study, Lc. garvieae was found in both male and female octopuses. Eventually, $L c$. garvieae and Lc. formosensis isolated from red octopus (Octopus maya) were detected as histamine producing strains. They represented $6 \%$ of the total isolates (Gullian et al., 2018). E. faecium, E. hirae, E. faecalis and $E$. gallinarum were isolated from faeces of black sea urchin (Arbacia lixula), sea urchin (Paracentrotus lividus), purple sea urchin (Sphaerechinus granularis), and sea cucumber (Holothuria mammata and Holothuria sanctori). The investigation showed that $E$. faecium was obtained as the predominant enterococcal species in the faecal samples. The enterococci showed high resistance to ampicillin, tetracycline and ciprofloxacin (Marinho et al., 2013).

A study conducted by Boulares and colleagues (2011) showed that Lb. brevis and Leu. mesenteroides was found in cuttlefish. Lc. lactis, P. pentosaceus, $L b$. curvatus, Leu. mesenteroides, Lb. paracasei, and $L b$. brevis were found in the muscle and intestine of octopus. The composition of bacterial communities in the gut

Table 3. Lactic acid bacteria isolated from invertebrate marine organism

\begin{tabular}{|c|c|c|c|c|}
\hline Marine Organism & Location & Lactic Acid Bacteria & Part of Isolation & References \\
\hline $\begin{array}{l}\text { Sea cucumber } \\
\text { (Apostichopus }\end{array}$ & $\begin{array}{l}\text { Weihai, Shandong } \\
\text { Province, China }\end{array}$ & $\begin{array}{l}\text { Carnobacterium, Lactococcus, } \\
\text { Lactobacillus, Streptococcus }\end{array}$ & GIT & $\begin{array}{l}\text { Wang et al. } \\
\text { (2018) }\end{array}$ \\
\hline $\begin{array}{l}\text { Black sea urchin } \\
(\text { Arbacia lixula), Sea } \\
\text { urchin (Paracentrotus }\end{array}$ & $\begin{array}{l}\text { São Miguel, São } \\
\text { Jorge and Flores, } \\
\text { Portugal }\end{array}$ & $\begin{array}{l}\text { Enterococcus faecium, E. hirae, } \\
\text { E. faecalis and E. gallinarum }\end{array}$ & Feces & $\begin{array}{l}\text { Marinho et al. } \\
\text { (2013) }\end{array}$ \\
\hline
\end{tabular}

lividus), Purple sea

urchin (Sphaerechinus

granularis), Sea

cucumber (Holothurai

mammata and

\begin{tabular}{|c|c|c|c|c|}
\hline $\begin{array}{l}\text { Chilean octopus } \\
\text { (Octopus mimus Gould) }\end{array}$ & Antofagasta, Chile & Lactococcus garvieae & Crop and Intestine & $\begin{array}{l}\text { Iehata et al. } \\
\text { (2013) }\end{array}$ \\
\hline $\begin{array}{l}\text { Red octopus (Octopus } \\
\text { maya) }\end{array}$ & $\begin{array}{l}\text { State of Yucatan, } \\
\text { Mexico }\end{array}$ & $\begin{array}{l}\text { Lactococcus garvieae and Lc. } \\
\text { formosensis }\end{array}$ & Crop & $\begin{array}{l}\text { Gullian et al. } \\
\text { (2018) }\end{array}$ \\
\hline Octopus & Tunisia & $\begin{array}{l}\text { Lc. lactis, Pediococcus } \\
\text { pentosaceus, Lb. curvatus, } \\
\text { Leuconostoc mesenteroides, Lb. } \\
\text { paracasei, Lb. brevis }\end{array}$ & Muscle and intestine & $\begin{array}{l}\text { Boulares et al. } \\
\text { (2011) }\end{array}$ \\
\hline Cuttlefish & Tunisia & Leu. mesenteroides, Lb. brevis & Muscle and intestine & $\begin{array}{l}\text { Boulares et al. } \\
\text { (2011) }\end{array}$ \\
\hline
\end{tabular}

* E-Enterococcus, Lc. - Lactococcus, Lb. - Lactobacillus, Leu. - Leuconostoc, GIT - gastrointestinal tract 
content of sea cucumber $A$. japonicus and ambient sediments of two different habitats, $Z$. marina seagrass bed and rocky intertidal habitat was compared using Illumina Miseq high-throughput sequencing. It showed that Carnobacterium, Lactococcus, Lactobacillus, and Streptococcus were relatively more abundant from seagrass bed habitat samples. This suggested that the seagrass bed ecosystem could represent a better source of candidate probiotics which beneficial for the survival of A. japonicus (Wang et al., 2018).

\subsection{Marine mammal}

Table 4 shows LAB isolated from marine mammals. Firmicutes phylum was composed as the highest in the dugong's faecal content. The bacterial flora in the same group including manatee and dugong may be varied according to several factors such as species, age, habitat, eating habits, digestive tract and tract position. It was unclear whether the seasonal variation can be related to the variation of chemical composition and digestion potential, or changes of the physiological or digestion metabolism of the dugong that related to the ageing of the animal (Tsukinowa et al., 2008). Lactobacillus salivarius isolated from the gastric fluid of bottlenose dolphin (Tursiops truncatus) revealed the potential to suppress the proliferation of enteric pathogen and stimulate tumour necrosis factor production in mammalian myeloid cells. Novel Lactobacillus spp. was isolated and related to Leuconostoc ceti (Diaz et al., 2013).

Two unidentified cocci were also isolated from the dead seal and porpoise. Comparative 16S rRNA gene sequencing was performed to determine the phylogenetic affinities of the two unknown cocci. Both isolates were $100 \%$ identical to each other and from the phylogenetic and phenotypic distinctiveness of the unknown bacterium, it was proposed as Vagococcus fessus (Hoyles et al., 2000).

\subsection{Marine reptile}

Sample from faeces and cloaca were collected from hospitalized sea turtles and the intestine samples were taken from the dead sea turtles. LAB was obtained from the faeces, cloaca and intestine of sea turtles (Caretta caretta). Sample from faeces and cloaca were collected from hospitalized sea turtles and the intestine samples were taken from the dead sea turtles. The location of the sample taken was in Tuscany and Liguria, Italy. From taxonomic composition, it showed that $66 \%$ of Firmicutes dominated the faeces sample and $87 \%$ from the intestine samples. Vagococcus with $42.3 \%$ was the most presented bacteria in the intestine (Abdelrhman et al., 2016). To the best of our knowledge, there were very limited studies conducted on marine reptiles.

\section{Conclusion}

Lactic acid bacteria have been isolated from marine animals from various sites of the animal's part in different parts of the geographical area. Various species were isolated from marine animals with the most dominant genus from Lactobacillus. The bacteria inhabit mostly the GIT of the animals. LAB protect the host by producing bacteriocin to control the growth of pathogens. The resistance of LAB towards antibiotics could be useful in playing a role as a probiotic in the medical industry. LAB isolated from the marine environment has the benefits of resilience to temperature and salty environment compared to other LAB strains. However, to the best of our knowledge, there is a lack of study in the discovery of the potential of LAB from marine animals to industries. More studies should be conducted to get the potential LAB to be manipulated into commercial products.

\section{Conflict of interest}

The authors declare no conflict of interest.

Table 4. Lactic acid bacteria isolated from marine mammal

\begin{tabular}{lllll}
\hline \multicolumn{1}{c}{ Marine Organism } & \multicolumn{1}{c}{ Location } & Lactic Acid Bacteria & Part of Isolation & References \\
\hline $\begin{array}{l}\text { Dugong (Dugong } \\
\text { dugong) }\end{array}$ & Toba, Japan & Lc. lactis ssp. lactis & Feces & $\begin{array}{l}\text { Tsukinowa } \text { et } \\
\text { al. (2008) }\end{array}$ \\
\hline $\begin{array}{l}\text { Bottlenose dolphin } \\
\text { (Tursiops truncatus) }\end{array}$ & $\begin{array}{l}\text { San Diego Bay, } \\
\text { California }\end{array}$ & Lb. salivarius, & $\begin{array}{l}\text { Oral and rectal swabs, milk, and } \\
\text { gastric fluid }\end{array}$ & $\begin{array}{l}\text { Diaz et al. } \\
(2013)\end{array}$ \\
\hline Seal & ni* & Vagococcus fessus sp. & Liver and kidney & Hoyles et al. \\
\hline Porpoise & ni* & Vagococcus fessus sp. & Peritoneum, spleen, kidney, & Hoyles et al. \\
& & & $\begin{array}{l}\text { liver, lung, brain, placenta and } \\
\text { small intestine. }\end{array}$ & $(2000)$ \\
\hline
\end{tabular}

* ni - no further information, Lb. - Lactobacillus, Lc. - Lactococcus 


\section{References}

Abdelrhman, K.F.A., Bacci, G., Mancusi, C., Mengoni, A., Serena, F. and Ugolini, A. (2016). A First Insight into the Gut Microbiota of the Sea Turtle Caretta caretta. Frontiers in Microbiology, 7, 1060. https:// doi.org/10.3389/fmicb.2016.01060

Al Bulushi, I.M., Poole, S.E., Barlow, R., Deeth, H.C. and Dykes, G.A. (2010). Speciation of GramPositive Bacteria in Fresh and Ambient-Stored SubTropical Marine Fish. International Journal of Food Microbiology, 138(1-2), 32-38. https:// doi.org/10.1016/j.ijfoodmicro.2009.11.021

Alonso, S., Castro, M.C., Berdasco, M., Banda, I.G., Moreno-Ventas, X. and Rojas, A.H. (2018). Isolation and Partial Characterization of Lactic Acid Bacteria from the Gut Microbiota of Marine Fishes for Potential Application as Probiotics in Aquaculture. Probiotics and Antimicrobial Proteins, 11(2), 569579. https://doi.org/10.1007/s12602-018-9439-2

Anup. (2018). Lactobacillus: Pathogenesis, Diseases, Diagnosis, Prevention. Retrieved on December 27, 2019 from Bioscience notes website: http:// www.biosciencenotes.com/lactobacilluspathogenesis-diseases-diagnosis-prevention/

Azahar, N.Z., Iehata, S., Fadhil, F., Bulbul, M. and Kader, M.A. (2018). Antimicrobial Activities of Lactic Acid Bacteria Isolated from Malaysian Prawn, Macrobrachium rosenbergii. Journal of Environmental Biology, 39(5), 821-824. https:// doi.org/10.22438/jeb/39/5(SI)/13

Azat, R., Liu, Y., Li, W., Kayir, A., Lin, D.B., Zhou, W.W. and Zheng, X.D. (2016) Probiotic Properties of Lactic Acid Bacteria Isolated from Traditionally Fermented Xinjiang Cheese. Journal of Zhejiang University-Science $B$ (Biomedicine and Biotechnology), 17(8), 597-609. https:// doi.org/10.1631/jzus.B1500250

Bahmani, Z., Rezaei, M., Hosseini, S.V., Hosseini, S.F., Alishahi, A., Ahmad, M. and Regenstein, J.M. (2014). Effect of Delayed Icing on the Microbiological, Chemical and Sensory Properties of Caspian Sea Golden Grey Mullet (Liza aurata). Journal of Aquatic Food Product Technology, 23(6), 542-551.

https:// doi.org/10.1080/10498850.2012.731677

Belfiore, C., Bjorkroth, J., Vihavainen, E., Raya, R. and Vignolo, G. (2010). Characterization of Leuconostoc strains Isolated from Fresh Anchovy (Engraulis anchoita). The Journal of General and Applied Microbiology, 56(2), 175-180. https:// doi.org/10.2323/jgam.56.175

Bennani, S., Mchiouer, K., Rokni, Y. and Meziane, M.
(2017). Characterisation and Identification of Lactic Acid Bacteria Isolated from Morrocan Raw Cow's Milk. Journal of Materials and Environmental Sciences, 8(S), 4934-4944

Bintsis, T. (2018). Lactic Acid Bacteria: Their Applications in Foods. Journal of Bacteriology and Mycology Open Access, 6(2), 89-94. https:// doi.org/10.15406/jbmoa.2018.06.00182

Boulares, M., Mejri, L. and Hassouna, M. (2011). Study of the Microbial Ecology of Wild and Aquacultured Tunisian Fresh Fish. Journal of Food Protection, 74 (10), 1762-1768. https://doi.org/10.4315/0362028X.JFP-11-057

Buntin, N., Chanthachum, S. and Hongpattarakere, T. (2008) Screening of Lactic Acid Bacteria from Gastrointestinal Tracts of Marine Fish for Their Potential Use as Probiotics. Songklanakarin Journal of Science and Technology, 30(1), 141-148

Cadwallader, K.R. and Singh, T.K. (2009). Flavours and Off-Flavours in Milk and Dairy Products. In McSweeney, P. and Fox, P. (Eds.) Advanced Dairy Chemistry, p. 631-690. New York, USA: Springer. https://doi.org/10.1007/978-0-387-84865-5_14

Cochran, J.K. (2014). Biological Oceanography. Reference Module in Earth Systems and Environmental Sciences. Elsevier E-Book. https:// doi.org/10.1016/b978-0-12-409548-9.09153-3

Dehler, C.E., Secombes, C.J. and Martin, S.A.M. (2017). Environmental and Physiological Factors Shape the Gut Microbiota of Atlantic Salmon Parr (Salmo salar L.). Aquaculture, 467, 149-157. https:// doi.org/10.1016/j.aquaculture.2016.07.017

Diaz, M.A., Bik, E.M., Carlin, K.P., Venn-Watson, S.K., Jensen, E.D., Jones, S.E., Gaston, E.P., Relman, D.A. and Versalovic, J. (2013). Identification of Lactobacillus Strains with Probiotics Features from the Bottlenose Dolphin (Tursiops truncatus). Journal of Applied Microbiology, 115(4), 1037-1051. https:// doi.org/10.1111/jam.12305

Florou-Paneri, P., Christaki, E. and Bonos, E. (2013). In Kongo, J.M. (Ed.) Lactic Acid Bacteria as Source of Functional Ingredients. InTech Open E-Book. https://doi.org/10.5772/47766

Gänzle, M.G. (2015). Lactic Metabolism Revisited: Metabolism of Lactic Acid Bacteria in Food Fermentations and Food Spoilage. Current Opinion in Food Science, 2, 106-117. https://doi.org/10.1016/ j.cofs.2015.03.001

Ghanbari, M., Jami, M., Kneifel, W. and Domig, K.J. (2013). Antimicrobial Activity and Partial Characterization of Bacteriocins Produced by Lactobacilli Isolated from Sturgeon Fish. Food 
Control, 32(2), 379-385. https://doi.org/10.1016/ j.foodcont.1012.12.024

Ghosh, A.S., Selvan, D.G., Neethu, C.S., Saramma, A.V. and Hatha, A.A.M. (2014). Diversity and Antimicrobial Activity of Lactic Acid Bacteria from the Gut of Marine Fish Rastrelliger kanagurta against Fish, Shrimp and Human Pathogens. Journal of the Marine Biological Association of India, 55(2), 22-27.

jmbai.2013.55.2.01778-04

Gullian K.M., Delgadillo Diaz, M. and Sanchez Soliz, M. (2018). Molecular Characterization of HistamineProducing Psychrotrophic Bacteria Isolated from Red Octopus (Octopus maya) in Refrigerated Storage. High-Throughput, 7(3), 25. https:// doi.org/10.3390/ht7030025

Gupta, R., Jeevaratnam, K. and Fatima, A. (2018). Lactic Acid Bacteria: Probiotic Characteristics, Selection Criteria, and Its Role in Human Health. Journal of Emerging Technologies and Innovative Research, 5 (10), 411-424

Hayek, S.A. and Ibrahim, S.A. (2013). Current Limitations and Challenges with Lactic Acid Bacteria: A Review. Food and Nutrition Science, 4 (11), 73-87. https://doi.org/10.4236/ fns.2013.411A010

Hoyles, L., Lawson, P.A., Foster, G., Falsen, E., Ohlen, M., Grainger, J.M. and Collins, M.D. (2000). Vagococcus fessus sp. nov., isolated from a seal and a harbor porpoise. International Journal of Systematic and Evolutionary Microbiology, 50(3), 1151-1154. https://doi.org/10.1099/00207713-50-31151

Iehata, S., Valenzuela, F. and Riquelme, C. (2013). Analysis of Bacterial Community and Bacterial Nutritional Enzyme Activity Associated with the Digestive Tract of Wild Chilean Octopus (Octopus mimus Gould, 1852). Aquaculture Research, 46(4), 861-873. https://doi.org/10.1111/are.12240

Itoi, S., Uchida, J. and Takanashi, S., Narita, T., Abe, K., Naya, S. and Sugita, H. (2013). The Clam Meretrix lamarckii (Bivalvia: Veneridae) is a Rich Respiratory of Marine Lactic Acid Bacteria Strains. Annals of Microbiology, 64(3), 1267-1274. https:// doi.org/10.1007/s13213-013-0771-1

Kang, C.H., Gu, T. and So, J.S. (2017). Possible Probiotic Lactic Acid Bacteria Isolated from Oysters (Crassostrea gigas). Probiotics and Antimicrobial Proteins, 10(4), 728-739. https://doi.org/10.1007/ s12602-01709315-5

Kang, C.H., Shin, Y.J., Kim, Y.G. and So, J.S. (2016). Isolation of Lactobacillus Strains from Shellfish for Their Potential Use as Probiotic. Biotechnology and
Bioprocess Engineering, 21(1), 46-52. https:// doi.org/10.1007/s122257-015-0518-x

Kanmani, P., Kumar, R.S., Yuvaraj, N., Paari, K.A., Pattukumar, V. and Arul, V. (2010). First Identification of a Novel Probiotic Bacterium Streptococcus phocae and Its Beneficial Role in Diseases Control. Journal of International Dental and Medical Research, 3(1), 45-51

Kennedy, J. (2019). Marine Life Definition and Examples. Retrieved on March 18, 2020 from Thoughtco Website: www.thoughtco.com/marinelife-defintion-and-examples-2291890

Kim, D.H. and Kim, D.Y. (2013). Microbial Diversity in the Intestine of Olive Flounder (Paralichthys olivaceus). Aquacultures, 414-415, 103-108. https:// doi.org/10.1016/j.aquaculture.2013.06.008

Kim, S.Y., Lee, M.J., Lee, J.J., Choi, S.H. and Kim, B.S. (2017). Analysis of Microbiota of the Swimming Crab (Portunus trituberculatus) in South Korea to Identify Risk Markers for Foodborne Illness. LWT Food Science and Technology, 86, 483-491. https:// doi.org/10.1016/j.lwt.2017.08.043

Kongnum, K. and Hongpattarakere, T. (2012). Effect of Lactobacillus plantarum Isolated from Digestive Tract of Wild Shrimp on Growth and Survival of White Shrimp (Litopenaeus vannamei) Challanged with Vibrio harveyi. Fish and Shellfish Immunology, 32(1), 170-177. https://doi.org/10.1016/ j.fsi.2011.11.008

Lee, H I., Kim, M.H., Kim, K.Y. and So, J.S. (2010). Screening and Selection of Stress Resistant Lactobacillus spp. Isolated from the Marine Oyster (Crassostrea gigas). Anaerobe, 16(5), 522-526. https://doi.org/10.1016/j.anaerobe.2010.07002

Maeda, M., Shibata, A., Biswas, G., Korenaga, H., Kono, T., Itami, T. and Sakai, M. (2014). Isolation of Lactic Acid Bacteria from Kuruma Shrimp (Marsupenaeus japonicus) Intestine and Assessment of Immunomodulatory Role of a Selected Strain as Probiotic. Marine Biotechnology, 16(2), 181-192. https://doi.org/10.1007/s10126-013-9532-1

Marinho, C., Silva, N., Pombo, S., Santos, T., Monteiro, R., Goncalves, A., Micael, J., Rodrigues, P., Costa, A.C., Igrejas, G. and Poeta, P. (2013). Echinoderms from Azores Islands: An Unexpected Source of Antibiotic Resistant Enterococcus spp. and Escherichia coli Isolates. Marine Pollution Bulletin, 69(1-2), 122-127. https://doi.org/10.1016/ j.marpolbul.2013.01.017

Masuda, M., Ide, M., Utsumi, H., Niiro, T., Shimamura, Y. and Murata, M. (2014). Production Potency of Folate, Vitamin B12, and Thiamine by Lactic Acid Bacteria Isolated from Japanese Pickles. Bioscience, 
Biotechnology, and Biochemistry, 76(11), 20612067. https://doi.org/10.1271/bbb.120414

Merrifield, D.L., Balcazar, J.L., Daniels, C., Zhou, Z., Carnevalli, O., Sun, Y.Z., Hoseinifar, S.H. and Ringo, E. (2014). Indigenous Lactic Acid Bacteria in Fish and Crustaceans. In Merrifield, D. and Ringo, E. (Eds.) Aquaculture Nutrition: Gut Health, Probiotics and Prebiotics, p. 128-168. United Kingdom: John Wiley and Sons. https:// doi.org/10.1002/9781118887263.ch6

Migaw, S., Ghrairi, T., Belguesmia, Y., Choiset, Y., Berjeaud, J.M., Chobert, J.M., Hani, K. and Haertle, T. (2013). Diversity of Bacteriocinogenic Lactic Acid Bacteria Isolated from Mediterranean Fish Viscera. World Journal of Microbiology and Biotechnology, 30(4), 1207-1217. https:// doi.org/10.1007/s11274-013-1535-6

Moosavi-Nasab, M., Abedi, E., Moosavi-Nasab, S. and Eskandari, M.H. (2014). Inhibitory Effect of Isolated Lactic Acid Bacteria from Scomberomorus commerson Intestines and Their Bacteriocin on Listeria innocua, Iran Agriculture Research, 33(1), 43-52. https://doi.org/10.22099/IAR.2014.2380

Mozzi, F. (2016). Lactic Acid Bacteria. Encyclopedia of Food and Health, p. 501-508. Reference Module in Food Science. Elsevier E-Book. https:// doi.org/10.1016/B978-0-12-384947-2.00414-1

Nguyen, V.D., Pham, T.T., Nguyen, T.H.H., Nguyen, T.T.X. and Hoj, L. (2014). Screening of Marine Bacteria with Bacteriocin-like Activities and Probiotic Potential for Ornate Spiny Lobster (Panulirus ornatus) juveniles. Fish and Shellfish Immunology, 40(1), 49-60. https://doi.org/10.1016/ j.fsi.2014.06.017

O'Bryan, C.A., Crandall, P.G., Ricke, S.C. and Ndahetuye, J.B. (2015). Lactic Acid Bacteria (LAB) as Antimicrobials in Food Products: Types and Mechanisms of Action. In Taylor, T.M. (Ed.) Handbook of Natural Antimicrobials for Food Safety and Quality, p. 117-136. USA: Academic Press. https://doi.org/10.1016/b978-1-78242-034-7.00006-2

Poffo, F. and Silva, M.A.C. (2011). Caracterização taxonômica e fisiológica de bactérias ácido-láticas isoladas de pescado marinho. Ciência e Tecnologia de Alimentos, 31(2), 303-307. https:// doi.org/10.1590/s0101-20612011000200004

Quinto, E.J., Jimenez, P., Caro, I., Tejero, J., Mateo, J. and Girbes, T. (2014). Probiotic Lactic Acid Bacteria: A Review. Food and Nutrition Science, 5 (18), 1765-1775. https://doi.org/10.4236/ fns. 2014.518190

Ringo, E., Hoseinifar, S.H., Ghosh, K., Doan, H.V.,
Beck, B.R. and Song, S.K. (2018). Lactic Acid Bacteria in Finfish - An Update. Frontiers in Microbiology, 9, e01818. https://doi.org/10.3389/ fmicb.2018.01818

Rungrassamee, W., Klanchui, A., Maibunkaew, S., Chaiyapechara, S., Jiravanichpaisal, P. and Karoonuthaisiri, N. (2014). Characterization of Intestinal Bacterial in Wild and Domesticated Adult Black Tiger Shrimp (Penaeus monodon). PLoS ONE 9(3), e91853. https://doi.org/10.1371/ journal.pone.0091853

Russo, P., Peña, N., de Chiara, M.L.V., Amodio, M.L., Colelli, G. and Spano, G. (2015). Probiotic Lactic Acid Bacteria for the Production of Multifunctional Fresh-Cut Cantaloupe. Food Research International, 77(Part 4), 762-772. https://doi.org/10.1016/ j.foodres.2015.08.033

Salas-Leiva, J., Opazo, R., Remond, C., Uribe, E., Velez, A. and Romero, J. (2017). Characterization of the Intestinal Microbiota of Wild-Caught and Farmed Fine Flounder (Paralichthys adspersus). Latin American Journal of Aquatic Research, 45(2), 370378. https://dx.doi.org/10.3856/vol45-issue2-fulltext12

Sarkono, Faturrahman and Sofyan, Y. (2010). Isolation and Identification of Lactic Acid Bacteria from Abalone (Haliotis asinine) as a Potential Candidate of Probiotic. Nusantara Bioscience, 2(1), 38-42. https://doi.org/10.13057/nusbiosci/n020106

Sha, Y., Liu, M., Wang, B., Jiang, K., Sun, G. and Wang, L. (2016). Gut Bacterial Diversity of Farmed Sea Cucumbers Apostichopus japonicus with Different Growth Rates. Microbiology, 85(1), 109-115. https:// doi.org/10.1134/S0026261716010112

Svanevik, C.S. and Lunestad, B.T. (2011). Characterisation of the Microbiota of Atlantic Mackerel (Scomber scombrus). International Journal of Food Microbiology, 151(2), 164-170. https://doi.org/10.1016/j.ijfoodmicro.2011.08.016

Talpur, A.D., Memon, A.J., Khan, M.I., Ikhwanuddin, M., Danish Daniel, M.M. and Abol-Munafi, A.B. (2012). Isolation and Screening of Lactic Acid Bacteria from the Gut of Blue Swimming Crab, $P$. pelagus, an In Vitro Inhibition Assay and Small Scale In Vivo Model for Validation of Isolates as Probiotics. Journal of Fisheries and Aquatic Science, 7(1), 1-28. https://doi.org/10.3923/jfas.2012.1.28

Tsukinowa, E., Karita, S., Asano, S., Wakai, Y., Oka, Y., Furuta, M. and Goto, M. (2008). Fecal Microbiota of a Dugong (Dugong dugong) in Captivity of Toba Aquarium. The Journal of General and Applied Microbiology, 54(1), 25-38. https://doi.org/10.2323/ jgam.54.25 
Uaboi-Egbenni, P.O., Okolie, P.N., Famuyiwa, O. and Teniola, O. (2010). The Significance of Pathogenic Bacteria in the Gut of Swimming Crab, Callinectes sp Obtained from Lagos Lagoon and Market Samples Stored at Freezer Temperature $\left(0^{\circ} \mathrm{C}\right)$. Pakistan Journal of Nutrition, 9(4), 398-403

Ucko, M. and Colorni, A. (2014). Infections by Lactic Acid Bacteria in Marine Fish from Southern Israel (Red Sea): New Records. The Israeli Journal of Aquaculture-Bamidgeh, 66, 1- 11.

Wang, Q., Zhang, X., Chen, M., Li, W. and Zhang, P. (2018). Comparison of Intestinal Microbiota and Activities of Digestive and Immune-Related Enzymes of Sea Cucumber Apostichopus japonicus in Two Habitats. Chinese Journal of Oceanology and Limnology, 36(3), 990-1001. https:// doi.org/10.1007/s00343-018-7075-z

Wei, H., Wang, H., Tang, L., Mu, C., Ye, C., Chen, L. and Wang, C. (2019). High-throughput Sequencing Reveals the Core Gut Microbiota of the Mud Crab (Scylla paramamosain) in Different Coastal Regions of Southern China. BMC Genomics, 20(1), 829. https://doi.org/10.1186/s12864-019-6219-7

Xu, S., Liu, T., Radji, C.A.I., Yang, J. and Chen, L. (2016). Isolation, Identification, and Evaluation of New Lactic Acid Bacteria Strains with Both Cellular Antioxidant and Bile Salt Hydrolase Activities In Vitro. Journal of Food Protection, 79(11), 19191928. https://doi.org/10.4315/0362-028X.JFP-16096

Yang, Q., Lu, Y., Zhang, M., Gong, Y., Li, Z., Tran, N. T., He, Y., Zhu, C., Lu, Y., Zhang, Y. and Li, S. (2019). Lactic Acid Bacteria, Enterococcus faecalis Y17 and Pediococcus pentosaceus G11, Improved Growth Performance, and Immunity of Mud Crab (Scylla paramamosain). Fish and Shellfish Immunology, 93, 135-143. https://doi.org/10.1016/ j.fsi.2019.07.050 